\title{
Utilization, trust and satisfaction with health care in adult sickle cell disease patients
}

\author{
Jacquelyn Baskin ${ }^{1}$, Anne Nord ${ }^{2}$, Dawn Canada ${ }^{2}$, Kelly Russell ${ }^{2}$, Payal Shah ${ }^{2}$, Thomas \\ Coates $^{2}$, and Arch Mainous ${ }^{3}$ \\ ${ }^{1}$ University of North Carolina System \\ ${ }^{2}$ Children's Hospital Los Angeles Children's Center for Cancer and Blood Diseases \\ ${ }^{3}$ University of Florida Department of Community Health and Family Medicine
}

July 23,2020

\begin{abstract}
Introduction: Transition from pediatric to adult care for emerging adults with sickle cell disease (SCD) has been challenging due to limited availability of experienced adult providers and patient difficulty navigating the adult health care system. The purpose of this study was to determine among adults with SCD, healthcare utilization and their trust and satisfaction with their health care provider. Methods: We surveyed adult patients greater than 21 years old with SCD previously transitioned from Children's Hospital Los Angeles. Assessments of provider trust and satisfaction were conducted along with health care utilization and the transition experience. Results: Of 31 participants, $61 \%$ and $68 \%$ identified having an adult primary care provider $(\mathrm{PCP})$ and adult hematologist respectively. Increased satisfaction with care was associated with increased trust in the adult hematologist $(\mathrm{r}=0.72 \mathrm{p}<0.001)$ and $\mathrm{PCP}(\mathrm{r}=0.76 \mathrm{p}=0.001)$ and improved communication $(\mathrm{p}<0.001)$. Trust in their hematologist was greater than PCP $(76.5$ vs $64.2, \mathrm{p}=0.058)$. For SCD complications, $65 \%$ of participants visited the ED, $80 \%$ of whom had negative experiences including sub-optimal pain management. Regarding transition experience, $55 \%$ felt unprepared for adult care. Discussion: More than $30 \%$ of adult SCD patients transferred out of pediatric care are not receiving regular hematology care for their SCD, resulting in fragmented medical care. Increased trust in their adult hematologist and clear communication are associated with higher levels of satisfaction with care. These findings will be utilized to develop a transition program to improve patient preparation and build on partnerships with adult providers to improve long-term outcomes.
\end{abstract}

\section{Introduction:}

Sickle cell disease (SCD) is one of the more common genetic conditions. It is characterized by intermittent exacerbation of vaso-occlusion by sickled red blood cells, leading to complications such as painful crisis, acute chest syndrome, stroke, and premature death. There are approximately 100,000 patients with this disease in the United States and as a result of its genetic predisposition, it disproportionately affects those of African and Hispanic descent. ${ }^{1,2}$ With newborn screening, vaccinations, prophylactic antibiotics, and hydroxyurea, the mortality in children with SCD decreased by $68 \%$ from 1983 to $2002 \cdot{ }^{3,4}$ As a result, more than $90 \%$ of patients are living to age 20 and the median survival has increased, now 58 years of age in patients with hemoglobin SS or S-beta thalassemia zero and 66 years of age in hemoglobin SC or S-beta thalassemia plus disease. ${ }^{5}$ However, studies have shown that morbidity and mortality increases dramatically in the 18-30 year old population, immediately after patients are transitioned from pediatric to adult centered care. ${ }^{6,7}$

Transition of care for patients with rare, genetic conditions, like SCD, whose manifestations start early in childhood has additional layers of complexity due to the limited availability of adult specialists with the necessary domain expertise to manage these patients. ${ }^{8-11}$ Adolescent and young adult (AYA) patients with SCD face a unique set of challenges including insurance issues, lack of transition skills and self-efficacy, 
compromised executive functioning, and stigmatization related to the need for narcotic analgesia. ${ }^{11-13} \mathrm{~A}$ majority of patients with SCD receive public insurance, which is associated with an increase in ED utilization, but fewer out-patient care visits, leading to fragmented long-term follow-up and inadequate comprehensive care. ${ }^{1,13-15}$ SCD patients are often discharged from pediatric practices to adult providers, frequently primary care providers who may be inexperienced or uncomfortable in managing this rare condition, which negatively impacts outcomes, health care utilization, and patient satisfaction with care. ${ }^{9,10,15,16}$ While patients expressed their perceived importance of transition, it has been demonstrated that many of them are poorly prepared for the transition process. ${ }^{17}$

Developing a transition plan that addresses key quality indicators such as improving communication between adult and pediatric providers, building trust between patients and new providers, and ensuring long-term follow-up are essential for improved outcomes for this population. ${ }^{18,19}$ It is clear that the research focus needs to shift towards new models of care to provide best practices regarding comprehensive care and patient outcomes for this high-risk population. It is unclear is how well SCD patients are currently transitioning to adult health care and how this process impacts the patient-provider relationship as well as future health care utilization. The purpose of this study is to determine the trust and satisfaction with current health care providers amongst a cohort of adult SCD patients. This study will provide a better understanding of their health care utilization practices and transition experience. This information can help direct the development of a transition program to improve this process and improve long-term patient outcomes.

\section{Methods:}

\section{Participant Identification:}

A quality improvement initiative was implemented amongst a cohort of adult SCD patients previously treated at Children's Hospital Los Angeles (CHLA) identified through the utilization of the hematology section database. All patients with the diagnosis of SCD that were over 21 years of age and had not had a medical visit at CHLA within the prior 12 months were eligible to participate. Participants were included regardless of current place of residence or current healthcare provider. IRB approval was obtained to allow for distribution of the information collected.

\section{Survey Development:}

A survey was developed to address patients' connection to adult providers, health care utilization including outpatient and inpatient visits, satisfaction and trust in adult health care providers, and information regarding their transition experience (Appendix 1). The Trust in Physician scale ${ }^{20}$ and portions of the Patient Satisfaction Questionnaire Short-Form (PSQ-18) ${ }^{21,22}$ were utilized to assess patient trust and satisfaction respectively. Questions regarding prescription and adherence to hydroxyurea, ED and hospital visits, and satisfaction with care provided in those visits were included. Lastly, subjects were asked to assess their satisfaction with perceived preparation for the transition process, ending with open-ended questions for feedback on how to improve this process. The completed survey was entered into Qualtrics to facilitate data collection and export for analysis.

\section{Participant Recruitment:}

Telephone communication was attempted with all patients who met the inclusion criteria. Telephone contact information was available within the CHLA hematology section database and the hospital's Electronic Medical Record system. Messages were left on voice-mail when available and multiple calls were attempted to all existing phone numbers until the patient was reached or it was determined that no current contact information was available. Emails and/or letters were sent to patients for whom telephone communication was not successful.

Statistical Analysis:

All categorical data was expressed as N (\%) and continuous data was expressed as mean (Standard Deviation). Dependent variables were assessed for normality, and the trust and communication scores among 
the hematologists were rank transformed. Linear regression was used to find the association between scores among PCP and hematologists. Wilcoxon rank sum test was used to detect the difference between PCP and hematologists' scores. A p-value of [?]0.05 was considered to be statistically significant. All tests were two-sided. The statistical software STATA/IC 14 was used for data analysis.

Results:

There were 140 patients with SCD identified in the hematology database that met the initial criteria for this survey. Of those patients, 93 patients could not be reached, 3 were too ill at the time to participate, 3 had a prior bone marrow transplant, and 7 patients were expired. A total of 34 potential participants agreed to participate in the survey. Three subjects were excluded, two because they only had a pediatric hematology provider at the time of the survey and one was later determined to have misinterpreted the survey. These data were removed considering it would potentially skew the results. Table 1 demonstrates the characteristics of the participants of this survey.

\section{Health Care Utilization:}

A summary of health care utilization can be found in Table 2. Of the evaluable surveys, $61 \%$ of participants indicated they are currently connected to an adult PCP and $68 \%$ are connected to an adult hematologist with $35 \%$ of participants having both an adult PCP and hematologist and only $6 \%$ not connected to any adult provider at the time of the survey. Seventy-nine percent have seen their PCP and $81 \%$ have seen their hematologist in the last 6 months. The patients connected to an adult hematologist were older than the patients that were not connected to an adult hematologist (mean difference 5 years, $\mathrm{p}=0.0002$ ). Type of SCD, location of participants' residence based on zip code, and time since last visit at CHLA were not found to be statistically significantly associated with having an adult provider, PCP and/or hematologist. Patients with both a PCP and a hematologist were more likely to have hydroxyurea prescribed than those with a PCP (91\% vs $37.5 \% \mathrm{p}=0.028)$ or hematologist ( $91 \%$ vs $40 \% \mathrm{p}=0.028$ ) alone.

Sixty-five percent of participants had visited the ED for a medical concern related to their SCD in the preceding 6 months, $30 \%$ of whom had at least 3 visits in that time-period. While only $40 \%$ of ED utilizers ranked some level of dissatisfaction with their experience, $80 \%$ provided negative statements regarding their ED care. Patients reported delays and sub-optimal dosing in pain management, lack of provider experience with SCD, and lack of trust of the ED provider in the patients' subjective report of pain symptoms as factors impacting their experience. Fifty-two percent of SCD subjects required inpatient hospitalization in the last 6 months with $25 \%$ admitted at least 3 times during that time frame. Of those that required hospitalization, $50 \%$ also had negative comments similar to those in the ED, although almost a third commented on positive experiences mostly related to satisfaction with the nursing care they received.

Connection to a PCP or a hematologist was not found to be significantly associated with decreased ED (Pearson $\mathrm{X}^{2}=0.23, \mathrm{p}=0.6$ and Pearson $\mathrm{X}^{2}=1.52, \mathrm{p}=0.22$ respectively) or hospital visits (Pearson $\mathrm{X}^{2}=2.50$, $\mathrm{p}=0.11$ and Pearson $\mathrm{X}^{2}=1.73, \mathrm{p}=0.19$ respectively). Moreover, neither the trust scores nor the general satisfaction scores for their adult providers were associated with differences in ED and hospital utilization ( $>0.1$ results not shown). Type of SCD was not associated with frequency of ED visits, but patients with either hemoglobin SS or S-beta zero thalassemia, the more severe types of SCD, were significantly associated with an increase in hospitalizations in the preceding 6 months (Pearson $\left.\mathrm{X}^{2}=4.04, \mathrm{p}=0.050\right)$.

\section{Satisfaction and Trust}

Participants indicated an increased level of general satisfaction with care was associated with a higher level of trust when evaluating care provided by the PCP $(\mathrm{r}=0.76, \mathrm{p}=0.001)$ or the adult hematologist $(\mathrm{r}=0.72, \mathrm{p}<0.001)$ (Figure 1A and 1B). Additionally, there were higher levels of general satisfaction associated with increased communication with either adult provider ( $\mathrm{PCP} r=0.78 \mathrm{p}<0.001$ and hematologist $\mathrm{r}=0.7$ $\mathrm{p}<0.001$ ) as well as improved access to care ( $\mathrm{PCP} \mathrm{r}=0.65 \mathrm{p}=0.003$ and hematologist $\mathrm{r}=0.49 \mathrm{p}=0.025)$. When assessing the relationship between trust and communication, it was noted that higher scores on the communication scale were associated with increased trust in the provider for both the PCP $(\mathrm{r}=0.67 \mathrm{p}=0.004)$ 
and hematologist $(\mathrm{r}=0.84 \mathrm{p}<0.001)$ (Figure $2 \mathrm{~A}$ and $2 \mathrm{~B}$ ) although no statistically significant association was noted between trust and access to care. Participants were noted to have higher levels of trust in the hematologist when compared to the PCP trending towards statistical significance, mean trust scores 76.5 vs $64.2,(\mathrm{p}=0.058)$. However, there was no difference in their sub-scores in general satisfaction, communication, or access to care when comparing these two groups.

Regarding transition experience, those participants that expressed that they felt extremely prepared for transition were noted to have higher levels of general satisfaction $(\mathrm{r}=0.6 \mathrm{p}=0.028)$ and trust $(\mathrm{r}=0.51 \mathrm{p}=0.05)$ with their PCP as well as higher levels of trust in their hematologist $(\mathrm{r}=0.49 \mathrm{p}=0.035)$. Participants' residential location impacted their perception of care from their hematologist. Those residing in the Metro and South Bay areas had significantly lower levels of both trust (median 57.96, $\mathrm{p}=0.004$ Metro and median 56.83, $\mathrm{p}=0.002$ South Bay) and general satisfaction (median 2.5, $\mathrm{p}=0.016$ Metro and median 2, $\mathrm{p}<0.001$ South Bay) when compared to the other areas in LA County.

One third of patients that indicated they are connected to a PCP had positive comments regarding their providers knowledge of their SCD and the care they received with that provider. Half of participants that indicated a connection to an adult hematologist had positive feedback including sentiments that their hematologist is a dedicated provider, knowledgeable about SCD, and resourceful in coordinating patients' medical care. However, about one third of patients were concerned about their hematologists' lack of knowledge regarding SCD, lack of trust in the medical care they received, and felt that the only service provided was medication refills.

\section{Transition Experience:}

Approximately half of participants, $52 \%$, expressed some level of dissatisfaction with their transition experience. While $45 \%$ felt at least somewhat prepared, the other $55 \%$ of participants did not feel prepared for the transition process. When asked for feedback regarding strategies to improve the transition process, the most common answers provided included facilitation of connection to an appropriate adult provider, with prior introduction, and to provide early interventions for transition preparation.

\section{Discussion:}

Even though almost one third of patients in this cohort were not connected to an adult hematologist, the overwhelming majority were connected to some type of an adult provider and had visited their provider in the prior 6 months. This represents a significant number of patients not connected to a hematologist, which is cause for concern considering PCPs lack of comfort and experience with the care of the patients with SCD. ${ }^{9,10,23,24}$ Prior studies indicated that increased distance to an SCD center was associated with increased ED visits but decreased in-patient hospitalizations which may results from fewer outpatient visits or lack of connection to an adult provider, although this association was not replicated in our study. ${ }^{14}$ Another difference is that the geographical location of this cohort is relatively equally dispersed throughout Los Angeles County (Figure 3) whereas prior studies completed in the Los Angeles area demonstrated higher densities of SCD patients in the Metro and Antelope Valley areas, also indicating this cohort may not be a completely unbiased representation of patients with SCD in this area. ${ }^{1,14}$

The rate of ED visits in this study is comparable to that reported in the literature for adults with SCD. Wolfson et al demonstrated $69 \%$ of the cohort had at least $1 \mathrm{ED}$ visit in the prior year. ${ }^{1,14} \mathrm{~A}$ more recent study indicates $50 \%$ of subjects have more than 3 visits and at least a third with 6 visits in a 12-month period, which is higher than our sample. ${ }^{25}$ There was a universal lack of satisfaction with ED care received by the participants in this study, particularly due to negative experiences related to suboptimal pain management and lack of providers' experience with SCD, leading to decreased trust in ED management. ${ }^{11,26-29}$ Based on patient feedback, it appears that patients might have improved ED or hospital visit experiences if they are connected to appropriate adult care. These negative ED experiences may also have a negative impact on patients' perception of their transition experience. ${ }^{26,28}$

Others have observed that $25-50 \%$ of adults with SCD required at least one hospitalization in the prior 
year as demonstrated here. ${ }^{30}$ However, it was surprising that the frequency of ED and hospital visits was not associated with lack of provider in this study. The literature supports a connection between decreased outpatient visits or poor comprehensive care with increased ED visits or hospitalizations. ${ }^{14,31-35}$ The lack of an adult provider or medical home contributes to fragmented, sub-optimal care, leading to increased need for ED care or increased complications leading to hospitalizations. One study documented the positive impact of a shared care model on frequency of hospitalizations indicating the need for both SCD directed comprehensive care in addition to general adult primary care. ${ }^{9}$

The association between trust and satisfaction as well as increased communication and access to care is consistent with other studies utilizing the Trust in Physician scale, where trust was associated with improved continuity with providers, adherence, and satisfaction with care. ${ }^{20,36}$ Studies in SCD patients specifically have also demonstrated this association between communication and trust, which are essential quality of care indicators associated with a successful transition of care. ${ }^{19,27}$ There was a trend towards increased trust in the hematologist, which result from the increased experience those providers have with these complex diagnoses. However, contrary to results from the PISCES study, there was no difference in satisfaction with care when comparing the PCP to the hematologist. ${ }^{13}$ There is a strong emphasis on trust because it is thought there is a compelling association between trust and adherence to medical advice. The results of our survey suggest that strategies for a successful transition include interventions to increase the trust between patients, PCP, and the adult hematologist by involving them in the transition plan.

More than $50 \%$ of participants were not satisfied with nor felt prepared for transition to adult care. Participant feedback indicated that facilitation of connection to an adult provider and the opportunity to meet them prior to transfer of care would be an important component to successful transition. This may improve the success of the transition of care and foster increased trust in the new provider, an essential aspect to transition. ${ }^{19,37}$ Additionally, increased perception of preparedness was associated with higher levels of satisfaction and trust in adult providers.

Participants suggested initiating transition education early to help prepare AYA patients for this process. The feedback obtained will inform the development of additional interventions incorporated into the ongoing development of a transition program.

\section{Limitations:}

The methods utilized to enroll patients in this study likely led to a selection bias considering the responses were only from those participants that the study team was able to communicate with directly utilizing only the contact information available in the hematology section database and electronic medical record. A large percentage of potentially eligible subjects were unable to be reached, which may indicate higher level of economic instability and potentially additional challenges with access to care and changes in contact information. This may have contributed to the discrepancy in results related to outpatient visits and it's lack of correlation with ED and hospital visits since this cohort may not represent patients that might be more transitory, leading to fewer outpatient visits with potentially higher frequency of ED visits. Additionally, this was a small cohort of patients from a single institution, so may not be an accurate representation of adult patients with SCD from other institutions or geographical locations.

\section{Conclusions:}

Although many of these patients are connected to ambulatory care, almost a third of patients are not connected to specialty care, which is sub-optimal and may impact their ability to receive comprehensive care. The role of trust and communication in providing a satisfactory patient experience is paramount and is an important quality of care indicator for successful transition of SCD patients. Strategies to optimize communication among patients, pediatric and adult providers as well as increase trust between patients and their new providers are essential to the development of a successful transition program. Interventions that will initiate early transition preparation and facilitate connection to appropriate adult providers, with potential opportunities to meet with providers prior to the final steps of transition, may increase trust in new providers and patient satisfaction and lead to a successful transition process. 
Conflict of Interest Statement : There were no conflicts of interest in the completion of this study or preparation of this manuscript

Acknowledgements: This research was made possible by philanthropic support of the Rauch Foundation and the Italian Catholic Federation.

\section{References}

1. Wolfson JA, Schrager SM, Coates TD, Kipke MD. Sickle-cell disease in California: A population-based description of emergency department utilization. Pediatric Blood $\&$ Cancer. 2011;56(3):413-419.

2. CDC Sickle Cell Disease home page - data and statistics - last accessed 2-18-2020. https://www.cdc.gov/ncbddd/sicklecell/data.html

3. Wojciechowski EA, Hurtig A, Dorn L. A natural history study of adolescents and young adults with sickle cell disease as they transfer to adult care: A need for case management services. Journal of Pediatric Nursing. 2002;17(1):18-27.

4. Yanni E, Grosse SD, Yang Q, Olney RS. Trends in Pediatric Sickle Cell Disease-Related Mortality in the United States, 1983-2002. The Journal of Pediatrics. 2009;154(4):541-545.

5. Elmariah H, Garrett ME, De Castro LM, et al. Factors associated with survival in a contemporary adult sickle cell disease cohort.American Journal of Hematology. 2014;89(5):530-535.

6. Shankar SM, Arbogast PG, Mitchel E, Cooper WO, Wang WC, Griffin MR. Medical care utilization and mortality in sickle cell disease: A population-based study. American Journal of Hematology.2005;80(4):262270.

7. Quinn CT, Rogers ZR, McCavit TL, Buchanan GR. Improved survival of children and adolescents with sickle cell disease. Blood.2010;115(17):3447-3452.

8. Lanzkron S, Sawicki GS, Hassell KL, Konstan MW, Liem RI, McColley SA. Transition to adulthood and adult health care for patients with sickle cell disease or cystic fibrosis: Current practices and research priorities. Journal of Clinical and Translational Science.2018;2(5):334-342.

9. Mainous AG, Rooks B, Tanner RJ, Carek PJ, Black V, Coates TD. Shared Care for Adults with Sickle Cell Disease: An Analysis of Care from Eight Health Systems. Journal of Clinical Medicine. 2019;8(8):1154.

10. Mainous AG, Tanner RJ, Harle CA, Baker R, Shokar NK, Hulihan MM. Attitudes toward Management of Sickle Cell Disease and Its Complications: A National Survey of Academic Family Physicians.Anemia. 2015;2015:1-6.

11. Debaun MR, Telfair J. Transition and Sickle Cell Disease.Pediatrics. 2012;130(5):926-935.

12. Treadwell M, Telfair J, Gibson RW, Johnson S, Osunkwo I. Transition from pediatric to adult care in sickle cell disease: Establishing evidence-based practice and directions for research. American Journal of Hematology. 2011;86(1):116-120.

13. Aisiku IP, Penberthy LT, Smith WR, et al. Patient satisfaction in specialized versus nonspecialized adult sickle cell care centers: the PiSCES study. J Natl Med Assoc. 2007;99(8):886-890.

14. Wolfson JA, Schrager SM, Khanna R, Coates TD, Kipke MD. Sickle cell disease in California: Sociodemographic predictors of emergency department utilization. Pediatric Blood \& Cancer.2012;58(1):66-73.

15. Brousseau DC. Acute Care Utilization and Rehospitalizations for Sickle Cell Disease. JAMA. 2010;303(13):1288.

16. Jordan L, Swerdlow P, Coates TD. Systematic review of transition from adolescent to adult care in patients with sickle cell disease.J Pediatr Hematol Oncol. 2013;35(3):165-169. 
17. McPherson M, Thaniel L, Minniti CP. Transition of patients with sickle cell disease from pediatric to adult care: Assessing patient readiness. Pediatric Blood E3 Cancer. 2009;52(7):838-841.

18. Wang CJ, Kavanagh PL, Little AA, Holliman JB, Sprinz PG. Quality-of-Care Indicators for Children With Sickle Cell Disease.Pediatrics 2011;128(3):484-493.

19. Sobota AE, Shah N, Mack JW. Development of quality indicators for transition from pediatric to adult care in sickle cell disease: A modified Delphi survey of adult providers. Pediatric Blood $\mathcal{G}$ Cancer. 2017;64(6):e26374.

20. Anderson LA, Dedrick RF. Development of the trust in physician scale: a measure to assess interpersonal trust in patient-physician relationships Psychol Rep. 1990;67(3 Pt 2):1091-1100.

21. Marshall GN and Hays R.D. The patient satisfaction questionnaire short form (PSQ-18). Santa Monica, CA: RAND Corporation; 1994.

22. Thayaparan AJ, Mahdi E. The Patient Satisfaction Questionnaire Short Form (PSQ-18) as an adaptable, reliable, and validated tool for use in various settings. Medical Education Online. 2013;18(1):21747.

23. Lunyera J, Jonassaint C, Jonassaint J, Shah N. Attitudes of Primary Care Physicians Toward Sickle Cell Disease Care, Guidelines, and Comanaging Hydroxyurea With a Specialist. Journal of Primary Care E Community Health. 2017;8(1):37-40.

24. Whiteman LN, Haywood C, Jr., Lanzkron S, Strouse JJ, Feldman L, Stewart RW. Primary Care Providers' Comfort Levels in Caring for Patients with Sickle Cell Disease. South Med J.2015;108(9):531-536.

25. Lanzkron S, Little J, Field J, et al. Increased acute care utilization in a prospective cohort of adults with sickle cell disease.Blood Advances. 2018;2(18):2412-2417.

26. Sobota A, Umeh E, Mack J. Young Adult Perspectives on a Successful Transition from Pediatric to Adult Care in Sickle Cell Disease.Journal of Hematology Research. 2016;2(1):17-24.

27. Haywood C, Lanzkron S, Bediako S, et al. Perceived Discrimination, Patient Trust, and Adherence to Medical Recommendations Among Persons with Sickle Cell Disease. Journal of General Internal Medicine.2014;29(12):1657-1662.

28. Porter JS, Wesley KM, Zhao MS, Rupff RJ, Hankins JS. Pediatric to Adult Care Transition: Perspectives of Young Adults With Sickle Cell Disease. Journal of Pediatric Psychology. 2017;42(9):1016-1027.

29. Puri Singh A, Haywood C, Beach MC, et al. Improving Emergency Providers' Attitudes Toward Sickle Cell Patients in Pain. Journal of Pain and Symptom Management. 2016;51(3):628-632.e623.

30. Shankar SM, Arbogast PG, Mitchel E, Ding H, Wang WC, Griffin MR. Impact of proximity to comprehensive sickle cell center on utilization of healthcare services among children with sickle cell disease.Pediatric Blood \& Cancer. 2008;50(1):66-71.

31. Blinder MA, Duh MS, Sasane M, Trahey A, Paley C, Vekeman F. Age-Related Emergency Department Reliance in Patients with Sickle Cell Disease. The Journal of Emergency Medicine.2015;49(4):513-522.e511.

32. Hemker BG, Brousseau DC, Yan K, Hoffmann RG, Panepinto JA. When children with sickle-cell disease become adults: Lack of outpatient care leads to increased use of the emergency department. American Journal of Hematology. 2011;86(10):863-865.

33. Okpala I, Thomas V, Westerdale N, et al. The comprehensive care of sickle cell disease. European Journal of Haematology.2002;68(3):157-162.

34. Cronin RM, Hankins JS, Byrd J, et al. Risk factors for hospitalizations and readmissions among individuals with sickle cell disease: results of a U.S. survey study. Hematology.2019;24(1):189-198. 
35. Blinder MA, Vekeman F, Sasane M, Trahey A, Paley C, Duh MS. Age-related treatment patterns in sickle cell disease patients and the associated sickle cell complications and healthcare costs.Pediatric Blood ES Cancer. 2013;60(5):828-835.

36. Thom DH, Ribisl KM, Stewart AL, Luke DA. Further validation and reliability testing of the Trust in Physician Scale. The Stanford Trust Study Physicians. Med Care. 1999;37(5):510-517.

37. Hankins JS, Osarogiagbon R, Adams-Graves P, et al. A Transition Pilot Program for Adolescents With Sickle Cell Disease. Journal of Pediatric Health Care. 2012;26(6):e45-e49.

Legends:

Figures:

1. Scatter plot of correlation between general satisfaction and trust in adult PCP and in adult hematologist

2. Trust vs Satisfaction - PCP

3. Trust vs Satisfaction - Hematologist

4. Scatter plot of correlation between trust and communication in adult PCP and in adult hematologist

5. Trust vs Communication - PCP

6. Trust vs Communication - Hematologist

7. Map of LA County with distribution of participants and location of adult providers

location of adult hematology providers

\section{Hosted file}

Table 1.docx available at https://authorea.com/users/345444/articles/471668-utilizationtrust-and-satisfaction-with-health-care-in-adult-sickle-cell-disease-patients

\section{Hosted file}

Table 2.docx available at https://authorea.com/users/345444/articles/471668-utilizationtrust-and-satisfaction-with-health-care-in-adult-sickle-cell-disease-patients

A

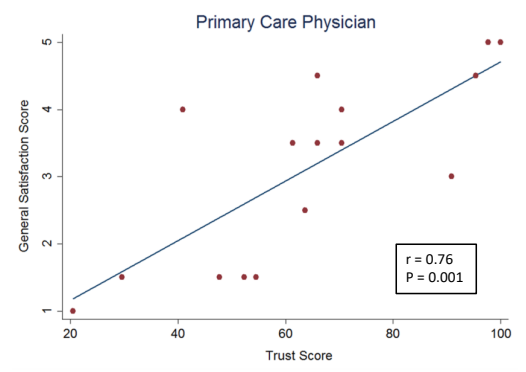

B

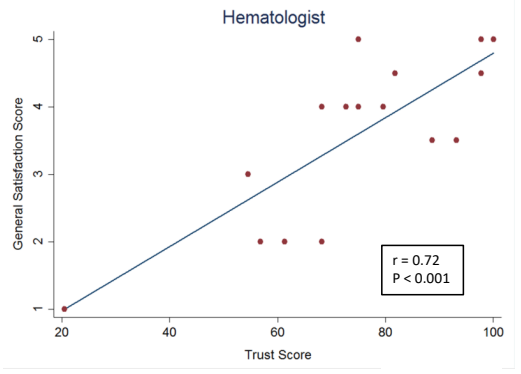


A

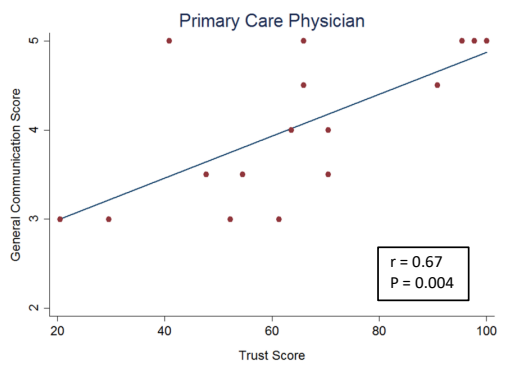

B

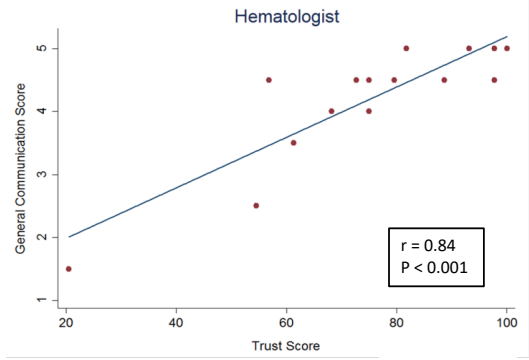

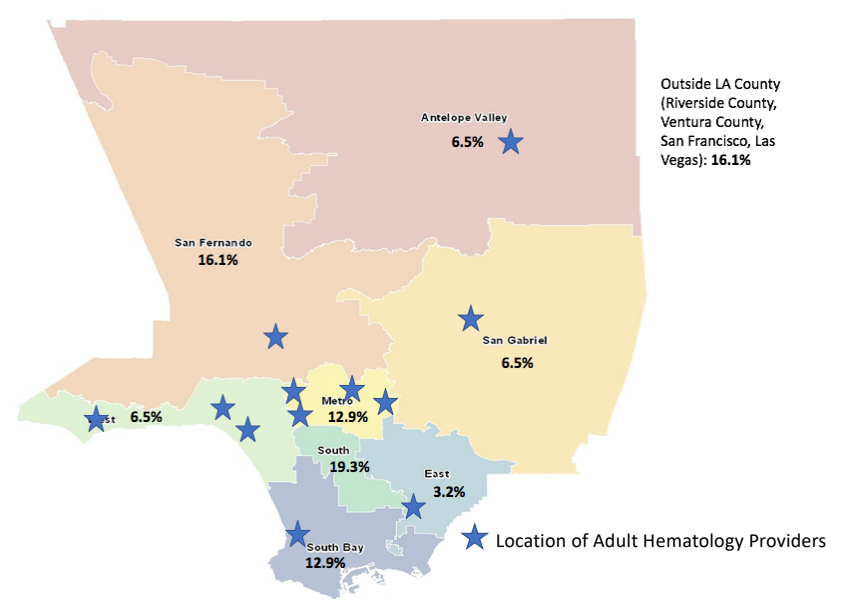

